\title{
SPECTRAL THEORY FOR SEMI-GROUPS OF LINEAR OPERATORS
}

BY

\author{
R. S. PHILLIPS
}

1. Introduction. In this paper various aspects of spectral theory for semigroups of linear transformations are investigated by means of an associated Banach algebra containing the semi-group of operators. This approach seems to be quite suited to the problems considered, and once the machinery has been set up the theorems follow a natural pattern.

The definitive work in this field is Hille's Functional analysis and semigroups $[6]\left({ }^{1}\right)$. By utilizing the theory of Banach algebras we have been able to extend many of Hille's results to a point which does not seem to be accessible by Laplace transform methods. We have also been able to obtain a new and direct proof of the Stone Theorem $[11 ; 1 ; 9]$ for locally compact abelian groups of unitary transformations. This proof makes extensive use of the representation theorem for commutative self-adjoint rings of operators on a Hilbert space and thus is able to avoid the use of positive definite functions.

We first sketch the theory of a Banach algebra $\subseteq$ consisting in essence of functions of bounded variation $\alpha$ on $(0, \infty)$ with convolution as their product. We then consider a commutative Banach algebra $\Re$ of linear bounded trans. formations containing the given semi-group of operators $T(t)$. By means of the mapping

$$
\Theta(\alpha)=\int_{0}^{\infty} T(t) d \alpha
$$

we are able to study the structure of the ring $\Re$ in terms of $\mathfrak{S}$. In particular the behavior of the multiplicative linear functionals on $\Theta(\alpha)$ is given in terms of the corresponding functionals on $\alpha$.

In section four we are concerned with semi-groups $T(t)$ uniformly continuous at the origin. Such a semi-group is completely characterized by the fact that it has a representation as a continuous function in the space of all maximal ideals $\mathfrak{M}^{\prime}=\left[m^{\prime}\right]$ which is of the form exp $\left[a\left(m^{\prime}\right) t\right]$. This in turn enables us to characterize the semi-groups with unbounded infinitesimal generators in terms of the form of their representation.

If we now define

$$
f_{\alpha}(\lambda)=\int_{0}^{\infty} \exp (\lambda t) d \alpha
$$

Presented to the Society, April 28, 1951; received by the editors March 8, 1951.

(1) Numbers in brackets refer to the references cited at the end of the paper. 
and denote the spectrum of an operator $B$ by $\Sigma(B)$, then for semi-groups with unbounded infinitesimal generator one obtains directly that $\Sigma[\Theta(\alpha)]$ $=f_{\alpha}[\Sigma(A)] \cup 0$ for $\alpha$ absolutely continuous. If we assume that $T(t)$ is uniformly continuous for $t \geqq c>0$, then for any $\alpha \in \subseteq$ we can show that $\Sigma[\Theta(\alpha)]$ $=f_{\alpha}[\Sigma(A)] \cup \alpha(0)$. Even more, this correspondence holds for the respective point, residual, and continuous parts of the spectra of $\Theta(\alpha)$ and $A$.

In section six we show that the indicator

$$
\sigma(\phi)=\lim _{r \rightarrow \infty} r^{-1} \log \|T[r \exp (i \phi)]\| \quad\left(\phi_{1}<\phi<\phi_{2}\right)
$$

is the function of support for the convex closed extension of the complex conjugate points to $\Sigma(A)$. Here we assume only that $T(\zeta)$ is analytic sufficiently far out in the sector $\left(\phi_{1}, \phi_{2}\right)$. Finally section seven is devoted to a new proof of the Stone Theorem and because of its independent interest this section is essentially self-contained.

In conclusion it should be pointed out that we have been unable to obtain any new results by these methods for the spectral theory of semi-groups of operators $T(t)$ which are merely strongly continuous for $t>0$.

2. The Banach algebra $\subseteq(\omega)$. It will be the purpose of this section to introduce a base space upon which we shall construct an operational calculus for the infinitesimal generator of any given semi-group of linear transformations. This space differs from the familiar Banach algebra of functions of bounded variation on $[0, \infty)$ with a convolution product only because of a weighting factor. Both A. Beurling [2] and I. Gelfand [5] have considered such algebras with continuous weighting functions on $(-\infty, \infty)$. It will be necessary for us to extend these results to Baire measurable weighting functions on $[0, \infty)$. Because of the close similarity with the continuous case we shall be very sketchy in our exposition.

We consider weighting functions $\exp [\omega(t)]$ with the following properties:

(a) $\omega(t)$ is a real-valued Baire measurable function defined on $[0, \infty)$,

(b) $\omega(0)=0 ; \quad \int_{0}^{1} \exp [\omega(t)] d t<\infty$,

(c) $\omega(t)$ is subadditive: $\omega\left(t_{1}+t_{2}\right) \leqq \omega\left(t_{1}\right)+\omega\left(t_{2}\right)$.

It can be shown (see [6, chap. 6]) that $\omega(t)$ is bounded in every finite subinterval $[\delta, 1 / \delta]$ and that

$$
\omega_{0}=\inf _{t>0} \omega(t) / t=\lim _{t \rightarrow \infty} \omega(t) / t .
$$

We now define $S(\omega)$ to be the set of all completely additive complexvalued set functions $\alpha(\sigma)$ on the sigma-field of Baire measurable subsets $\mathbb{B}$ of $[0, \infty)$ such that $\int_{0}^{\infty} \exp [\omega(t)]|d \alpha|<\infty$. The norm is given by 


$$
\|\alpha\|=\int_{0}^{\infty} \exp [\omega(t)]|d \alpha| .
$$

It is clear that $\subseteq(\omega)$ is a Banach space. In order to define the product $\gamma=\alpha \beta$ of two elements in $\subseteq(\omega)$; we consider the product measure $\bar{\gamma}$ defined in the smallest sigma-field $\mathfrak{B}_{2}$ generated by $\mathfrak{B} \times \mathfrak{B}$. For any $\sigma \in \mathfrak{B}$ we set

$$
\gamma(\sigma) \equiv \bar{\gamma}[(u, v) \mid u+v \in \sigma ; u, v \geqq 0] .
$$

This product is clearly commutative; with the help of the Fubini Theorem it becomes

$$
\gamma(\sigma)=\int_{0}^{\infty} \alpha(\sigma-u) d_{u} \beta=\int_{0}^{\infty} \beta(\sigma-u) d_{u} \alpha .
$$

It also follows from the Fubini Theorem that

$$
\begin{aligned}
\|\gamma\| & =\int_{0}^{\infty} \exp [\omega(s)]\left|d_{s} \gamma\right|=\int_{0}^{\infty} \int_{0}^{\infty} \exp [\omega(u+v)]\left|d_{u} \alpha\right|\left|d_{v} \beta\right| \\
& \leqq \int_{0}^{\infty} \int_{0}^{\infty} \exp [\omega(u)] \exp [\omega(v)]\left|d_{u} \alpha\right|\left|d_{v} \beta\right|=\|\alpha\| \cdot\|\beta\| .
\end{aligned}
$$

Finally we set

$$
e_{t}(\sigma)= \begin{cases}1 & \text { if } t \in \sigma \\ 0 & \text { otherwise }\end{cases}
$$

Then $e_{0}$ is the unit of our algebra and $e_{t} \alpha=\alpha(\sigma-t)$ is the translate of $\alpha(t$ units to the right); $\left\|e_{t}\right\|=\exp [\omega(t)]$. As defined, $\subseteq(\omega)$ is a commutative Banach algebra with unit.

Now let $\mathfrak{R}(\omega)$ be the absolutely continuous (relative to Lebesgue measure) elements of $\mathfrak{S}(\omega)$. Then $\mathfrak{R}(\omega)$ is an ideal in $\subseteq(\omega)$ and for each $\alpha \in \mathfrak{R}(\omega), e_{t} \alpha$ is a continuous function of $t$ (in the norm sense). Hence for $\alpha \in \mathcal{R}(\omega)$ we may interpret the convolution (4) as an abstract Lebesgue-Stieltjes integral of a continuous function in $\subseteq(\omega)$, namely,

$$
\gamma=\int_{0}^{\infty}\left(\alpha e_{t}\right) d_{t} \beta
$$

According to the Gelfand theory [3] of Banach algebras, each maximal ideal $m$ corresponds to a unique multiplicative linear functional $\mu_{m}$ such that

(a) $\mu_{m}(\alpha)=0$ if and onl $y$ if $\alpha \in m$,

(b) $\mu_{m}\left(e_{0}\right)=1$,

(c) $\mu_{m}(\alpha+\beta)=\mu_{m}(\alpha)+\mu_{m}(\beta) ; \mu_{m}(c \alpha)=c \mu_{m}(\alpha) ; \mu_{m}(\alpha \beta)=\mu_{m}(\alpha) \cdot \mu_{m}(\beta)$. 
The set of all maximal ideals $\mathfrak{M}$ can be topologized to form a compact Hausdorff space with a general neighborhood of $m_{0}$ defined by a finite set of $\alpha$ 's and $\epsilon>0$ to be $\left[m|| \alpha_{i}(m)-\alpha_{i}\left(m_{0}\right) \mid\langle\epsilon ; i=1, \cdots, n]\right.$. It is clear that in this topology the representation $\alpha(m)=\mu_{m}(\alpha)$ is a continuous function on $\mathfrak{M}$. Another consequence of the theory is that an $\alpha \in \mathfrak{S}(\omega)$ has an inverse in $\mathfrak{S}(\omega)$ if and only if $\alpha$ does not belong to any $m \in \mathfrak{M}$ (that is, if $\alpha(m)$ is never zero).

We shall distinguish between three types of maximal ideals:

(a) $\mathfrak{B}=$ all maximal ideals $m$ such that $\mathfrak{R}(\omega)$ is not contained in $m$.

(b) $3=$ the maximal ideal consisting of all $\alpha$ such that $\alpha(0)=0$.

(c) $\mathfrak{U}=\mathfrak{M}-(\mathfrak{B} \cup \mathfrak{Z})$.

THEOREM $2.1 \mathfrak{W}$ is homeomorphic with the complex numbers $\mathfrak{S}_{0}$ $\equiv\left[\lambda \mid R(\lambda) \leqq \omega_{0}\right]$. Under this correspondence $\mu_{m}(\alpha)=\int_{0}^{\infty} \exp (\lambda t) d \alpha$. $\mathfrak{W}$ is an open subset of $\mathfrak{M}$.

If $\lambda \in \mathfrak{C}_{0}$, then it is clear that $\mu(\alpha)=\int_{0}^{\infty} \exp (\lambda t) d \alpha$ exists by equations (2), (3) and defines a linear multiplicative functional on $\subseteq(\omega)$. Since $\mu(\alpha)$ does not vanish for all $\alpha \in \mathfrak{R}(\omega)$, it follows that $m_{\lambda}=[\alpha \mid \mu(\alpha)=0]$ belongs to $\mathfrak{B}$. On the other hand if $m \in \mathfrak{B}$, then there exists an $\alpha_{0} \in \mathcal{R}(\omega)$ such that $\mu_{m}\left(\alpha_{0}\right) \neq 0$. Now $\mu_{m}\left(\alpha_{0} e_{t}\right)=\mu_{m}\left(\alpha_{0}\right) \mu_{m}\left(e_{t}\right)$ is continuous in $t$ and hence $\mu_{m}\left(e_{t}\right) \rightarrow \mu_{m}\left(e_{0}\right)=1$ as $t \rightarrow 0$. Further $\mu_{m}\left(e_{t+s}\right)=\mu_{m}\left(e_{t}\right) \cdot \mu_{m}\left(e_{s}\right)$. It follows that there exists a complex number $\lambda$ such that $\mu_{m}\left(e_{t}\right)=\exp (\lambda t)$. By (2)

$$
R(\lambda)=t^{-1} \log |\exp (\lambda t)| \leqq \inf \left[t^{-1} \log \left\|e_{t}\right\|\right]=\omega_{0} .
$$

For any $\beta \in \subseteq(\omega)$, we have by (6)

$$
\begin{aligned}
\mu_{m}\left(\alpha_{0}\right) \cdot \mu_{m}(\beta) & =\mu_{m}\left(\alpha_{0} \beta\right)=\mu_{m}\left[\int_{0}^{\infty}\left(\alpha_{0} e_{t}\right) d_{t} \beta\right] \\
& =\int_{0}^{\infty} \mu_{m}\left(\alpha_{0} e_{t}\right) d_{t} \beta=\int_{0}^{\infty} \mu_{m}\left(\alpha_{0}\right) \exp (\lambda t) d_{t} \beta
\end{aligned}
$$

and hence $\mu_{m}(\beta)=\int_{0}^{\infty} \exp (\lambda t) d_{t} \beta$. Finally since $\exp (\lambda t)=\int_{0}^{\infty} \exp (\lambda s) d_{s} e_{t}$, it is clear that the correspondence between $m \in \mathfrak{B}$ and $\lambda \in \mathfrak{S}_{0}$ is one-to-one.

We next show that the correspondence is actually a homeomorphism. Now $\mu_{\lambda}(\alpha)=\int_{0}^{\infty} \exp (\lambda t) d \alpha$ is a continuous function of $\lambda$ on $\mathfrak{E}_{0}$ for each $\alpha \in \subseteq(\omega)$. Hence an arbitrary neighborhood in $\mathfrak{B}$, namely,

$$
\left[\lambda|| \mu_{\lambda}\left(\alpha_{i}\right)-\mu_{\lambda_{0}}\left(\alpha_{i}\right) \mid<\epsilon ; \lambda \in \mathfrak{E}_{0} ; i=1, \cdots, n\right]
$$

corresponds to an open subset of $\mathfrak{E}_{0}$. On the other hand, given a neighborhood $\mathfrak{B}=\left[\lambda|| \lambda-\lambda_{0} \mid<\epsilon, \lambda \in \mathfrak{S}_{0}\right]$ of $\lambda_{0}$ in $\mathfrak{E}_{0}$ we show that it contains a $\mathfrak{B}$ neighborhood. The real part of $\lambda$ is sufficiently limited by 


$$
\left|R(\lambda)-R\left(\lambda_{0}\right)\right|=|\log | \mu_{\lambda}\left(e_{1}\right)|-\log | \mu_{\lambda_{0}}\left(e_{1}\right)||<\epsilon / 2 ;
$$

in other words by $\left|\mu_{\lambda}\left(e_{1}\right)-\mu_{\lambda_{0}}\left(e_{1}\right)\right|<\left|\mu_{\lambda_{0}}\left(e_{1}\right)\right| \cdot \epsilon / 2$. Further given an $\alpha_{0} \in \mathcal{L}(\omega)$ such that $\mu_{\lambda_{0}}\left(\alpha_{0}\right) \neq 0$, it follows from the Riemann-Lebesgue Theorem that there exists an $N$ such that $\left|\mu_{\lambda}\left(\alpha_{0}\right)-\mu_{\lambda_{0}}\left(\alpha_{0}\right)\right|<\mu_{\lambda_{0}}\left(\alpha_{0}\right) / 2$ excludes all $\lambda \in \mathfrak{E}_{0}$ for which $|\Im(\lambda)|>N$. Finally we can exclude all $\lambda \in \mathcal{S}_{0}$ for which $\epsilon / 2 \leqq\left|\Im(\lambda)-J\left(\lambda_{0}\right)\right|$ $<N$ by the condition $\left|\mu_{\lambda}\left(e_{1 / N}\right)-\mu_{\lambda_{0}}\left(e_{1 / N}\right)\right|<\left|\mu_{\lambda_{0}}\left(e_{1 / N}\right)\right| \cdot \sin \epsilon / 2 N$. It remains to show that $\mathfrak{W}$ is an open subset of $\mathfrak{M}$ and this follows from the fact that $\mathfrak{W}$ is the union of the open sets $\left[m|| \mu_{m}(\alpha) \mid>0\right]$ where $\alpha$ ranges over $\mathbb{R}(\omega)$.

TheOREM 2.2. If $\mu_{0}$ corresponds to 3 , then $\mu_{0}(\alpha)=\alpha(0)$. Further $\mu_{0}$ is the only multiplicative linear functional such that $\mu\left(e_{t}\right)=0$ for some $t>0$.

Since $\alpha(0)+\beta(0)=(\alpha+\beta)(0), \alpha \beta(0)=\alpha(0) \cdot \beta(0),|\alpha(0)| \leqq\|\alpha\|$, and $e_{0}(0)$ $=1$, it follows that $\mu_{0}(\alpha)=\alpha(0)$ is a multiplicative linear functional and hence that $B$ is the corresponding maximal ideal. Suppose $\mu$ is a multiplicative linear functional and $\mu\left(e_{t_{0}}\right)=0\left(t_{0}>0\right)$. Since $\mu\left(e_{t+s}\right)=\mu\left(e_{t} e_{s}\right)=\mu\left(e_{t}\right) \cdot \mu\left(e_{s}\right)$, it follows that $\mu\left(e_{t}\right)=0$ for all $t \geqq t_{0}$. Further for any $t>0$ there is an $n$ such that $n t \geqq t_{0}$, and hence $\mu\left(e_{t}\right)=\left[\mu\left(e_{n t}\right)\right]^{1 / n}=0$. Finally let $\alpha \in \Im(\omega)$ and suppose $\alpha(0)=0$. We then set $I_{\delta}=[0, \delta]$ and define $\alpha_{\delta}(\sigma)=\alpha\left(\sigma-I_{\delta} \cap \sigma\right)$. Then $\lim _{\delta \rightarrow 0}\left\|\alpha_{\delta}-\alpha\right\|=0$. Further $\alpha_{\delta} e_{-\delta / 2} \in \Im(\omega)$ and $\alpha_{\delta}=\left(\alpha_{\delta} e_{-\delta / 2}\right) e_{\delta / 2}$. Hence $\mu\left(e_{\delta / 2}\right)=0$ implies that $\mu\left(\alpha_{\delta}\right)=0$ and by continuity that $\mu(\alpha)=0$. For arbitrary $\alpha \in \mathbb{S}(\omega)$, $\mu(\alpha)=\mu\left(\alpha-\alpha(0) e_{0}\right)+\mu\left(\alpha(0) e_{0}\right)=\alpha(0)$; hence $\mu=\mu_{0}$.

THEOREM 2.3. The multiplicative linear functional $\mu$ corresponds to $a$ maximal ideal in $\mathfrak{U}$ if and only if $\mu[\mathfrak{R}(\omega)]=0$ and $\mu\left(e_{t}\right)=\exp (\xi t) \chi(t)$ where $\xi \leqq \omega_{0}$ and $\chi(t)$ is a character of the real line.

It follows from the definition of $\mathfrak{U}$ and the previous two theorems that $\mu \in \mathfrak{U}$ if and only if $\mu[\mathcal{L}(\omega)]=0$ and $\mu\left(e_{t}\right)$ is never zero. Now $\mu\left(e_{0}\right)=1, \mu\left(e_{t+s}\right)$ $=\mu\left(e_{t}\right) \cdot \mu\left(e_{s}\right)$, and $\left|\mu\left(e_{t}\right)\right| \leqq\left\|e_{t}\right\|=\exp [\omega(t)]$, which is bounded in every finite interval $[\delta, 1 / \delta]$. By a well known theorem on linear functions, $\log \left|\mu\left(e_{t}\right)\right|=\xi t$. Further $\xi \leqq \inf \omega(t) / t=\omega_{0}$. Finally $\exp (-\xi t) \mu\left(e_{t}\right)$ is clearly a character of the real line. This character $\chi(t)$ may be either continuous or nonmeasurable.

If $\mu \in \mathfrak{U}$, it is not known how to express $\mu(\alpha)$ in terms of $\mu\left(e_{t}\right)$. However if we limit $\Im(\omega)$ to contain no singular functions, then for $\mu \in \mathfrak{U}$ we have $\mu(\alpha)=\sum_{t \geqq 0} \mu\left(e_{t}\right) \cdot \alpha(t)$. Also in this case it can be shown that $\mathfrak{W}$ is dense in $\mathfrak{M}$. This very important fact leads directly to an inversion theorem of the Wiener-Pitt type. However, since we shall not need this result we do not give the proof and merely remark that it is a consequence of the RiemannLebesgue Theorem and the Kronecker Theorem (see [5, pp. 61-62]).

We conclude this section with a characterization of nonmeasurable characters which we shall have occasion to use in section four.

LEмма 2.1. Let $\chi(t)$ be a character of the real line and set $\mathfrak{F}_{\tau}=[\chi(t) \mid 0 \leqq t \leqq \tau]$ 
and $\mathfrak{F}=\bigcap_{\tau>0} \overline{\mathfrak{F}}_{\tau}$. There are two alternatives: either $\mathfrak{F}=1$ in which case $\chi(t)$ is a continuous character and $\chi(t)=\exp (a t)$; or else $\mathfrak{F}$ contains the unit circle and $\chi(t)$ is nonmeasurable.

If $\mathfrak{F}$ consists only of the point 1 , then given $\epsilon>0$ there exists a $\delta>0$ such that $|\chi(t)-1|<\epsilon$ for all $t \in[0, \delta]$. Otherwise the closed interval on the unit circle $[|\exp (i \phi)-1| \geqq \epsilon]$ would contain points of $\chi(t)$ for arbitrarily small $t$ and hence would have points in common with $\mathfrak{F}$. Therefore $\chi(t)$ is continuous at the origin and of the form $\exp (a t)$.

On the other hand suppose $\exp \left(i \phi_{0}\right) \in \mathfrak{F}\left(0<\phi_{0}<2 \pi\right)$. Then there exists a sequence $t_{n} \rightarrow 0$ such that $\chi\left(t_{n}\right) \rightarrow \exp \left(i \phi_{0}\right)$ and hence $\chi\left(k t_{n}\right) \rightarrow \exp \left(i k \phi_{0}\right)$ so that $\exp \left(i k \phi_{0}\right)$ likewise belongs to $\mathfrak{F}$. Further if $\exp \left(i \phi_{1}\right)$ and $\exp \left(i \phi_{2}\right)$ belong to $\mathfrak{F}$, then similarly exp $\left[i\left(\phi_{1}+\phi_{2}\right)\right]$ belongs to $\mathfrak{F}$. Thus if $\mathfrak{F}$ is not dense on the unit circle it must contain a smallest argument $\phi_{0}$ and $\mathfrak{F}$ consists precisely of the points $\left[\exp \left(i k \phi_{0}\right) \mid k=1,2, \cdots, 2 \pi / \phi_{0}\right]$. Again there exists a sequence $t_{n} \rightarrow 0$ such that $\chi\left(t_{n}\right) \rightarrow \exp \left(i \phi_{0}\right)$. In this case $\chi\left(t_{n} / 2\right)$ will contain a subsequence which converges either to $\exp \left(i \phi_{0} / 2\right)$ or to $-\exp \left(i \phi_{0} / 2\right)$. Neither of these points is in the set $\left[\exp \left(i k \phi_{0}\right)\right]$ and hence $\mathfrak{F}$ must be dense in the unit circle. Since $\mathfrak{F}$ is closed it must consist of all points in the unit circle.

It should be remarked that there is nothing special about $t=0$ in the above lemma and that a similar result holds for any real $t$.

Before closing this section it would be well to point out that most of the results in this paper can be obtained without the use of $\mathfrak{S}(\omega)$. However, in proceeding as we do we are able to reduce part of the difficulty to the behavior of the relatively simple Banach algebra $\mathfrak{S}(\omega)$. Furthermore $\mathfrak{S}(\omega)$ will enter directly in the statement of some of our theorems.

3. Banach algebra associated with the semi-group of operators. Let $\mathfrak{X}$ be a complex Banach space, $\mathfrak{F}(\mathfrak{X})$ the bounded linear transformations on $\mathfrak{X}$ to itself, and let $T(t)$ be a semi-group (see [6]) of operators on $[0, \infty)$ to $\mathfrak{E}(\mathfrak{X})$ satisfying the following hypothesis.

Hypothesis $\mathrm{H}$.

(a) $T\left(t_{1}+t_{2}\right)=T\left(t_{1}\right) T\left(t_{2}\right)$, $t_{1}, t_{2} \geqq 0$,

$$
T(0)=I \text {, }
$$

(b) $T(t) x$ is strongly measurable,

(c) $\int_{0}^{1}\|T(t)\| d t<\infty$,

(d) $\lim _{\tau \rightarrow 0} \tau^{-1} \int_{0}^{\tau} T(t) x d t=x$ for all $x \in \mathfrak{X}$.

Postulate (H-b) implies that $T(t)$ is strongly continuous for $t>0$ and hence that $\|T(t)\|$ is lower semi-continuous (see [6] and [10]). It is therefore clear 
that the subadditive function,

$$
\omega(t)=\log \|T(t)\|
$$

satisfies the conditions (1) and may therefore be used to define a Banach algebra of the type $\mathfrak{S}(\omega)$.

Let $A$ be the infinitesimal generator of a semi-group $T(t)$ of type $(\mathrm{H})$. Then $A$ will be a closed linear operator, in general unbounded, with dense domain $\mathfrak{D}(A)$. Let $R(\lambda ; A)$ be the resolvent of $A$ in $\mathfrak{E}(\mathfrak{X})$ defined and holomorphic on the resolvent set for $A$, namely $\rho(A)$. On this set $R(\lambda ; A)$ satisfies the first resolvent equation

$$
R(\lambda ; A)-R(\zeta ; A)=(\zeta-\lambda) R(\lambda ; A) R(\zeta ; A)
$$

as well as the defining equations

$$
\begin{aligned}
& (\lambda I-A) R(\lambda ; A) x=x \\
& R(\lambda ; A)(\lambda I-A) x=x
\end{aligned}
$$$$
\text { for } x \in \mathfrak{X} \text {, }
$$$$
\text { for } x \in \mathfrak{D}(A) \text {. }
$$

For $R(\lambda)>\omega_{0}$

$$
R(\lambda ; A) x=\int_{0}^{\infty} \exp (-\lambda t) T(t) x d t .
$$

The spectrum of $A$, denoted by $\Sigma(A)$, is by definition the complementary set to $\rho(A)\left({ }^{2}\right)$. Among other things, we shall be concerned with the relationship between $\Sigma(A)$, the spectrum of $T(t)$, and rate of growth properties of $T(t)$.

Let $\mathfrak{A}_{1}$ consist of the operators

$$
\begin{gathered}
{[T(t) \mid t \geqq 0],} \\
{[R(\lambda ; T(t)) \mid t \geqq 0, \lambda \in \rho[T(t)]],} \\
{[R(\lambda ; A) \mid \lambda \in \rho(A)] .}
\end{gathered}
$$

We shall now show that $\mathfrak{A}_{1}$ is an abelian set. In the first place $T(t)$ leaves $\mathfrak{D}(A)$ invariant and for $x \in \mathfrak{D}(A)$ we have $T(t) A x=A T(t) x$. Hence by (11)

$$
\begin{aligned}
(\lambda I-A) T(t) R(\lambda ; A) x & =T(t)(\lambda I-A) R(\lambda ; A) x \\
& =T(t) x=(\lambda I-A) R(\lambda ; A) T(t) x
\end{aligned}
$$

for all $x \in \mathfrak{X}$. Operating on the first and last members of this series of equalities by $R(\lambda ; A)$ gives $R(\lambda ; A) T(t)=T(t) R(\lambda ; A)$. Finally since $R(\lambda ; T(t))$ is the inverse of $[\lambda I-T(t)]$, it commutes with everything that commutes with $T(t)$.

We now define $\Re_{1}$ to be the strong closure of the set of all polynomials in $\mathfrak{A}_{1}$. Then $\Re_{1}$ is an abelian subring of $\mathfrak{E}(\mathfrak{X})$. In fact let $\mathfrak{R}^{\prime}$ be any abelian subring of $\mathfrak{E}(\mathfrak{X})$ and let $\Re$ be its strong closure. Then if $A, B \in \Re$ it is clear that

(2) The point at infinity is never considered as belonging to $\Sigma(A)$, not even if $R(\lambda ; A)$ is singular at infinity. 
$a A$ and $A+B$ belong to $\Re$. Given $\left(x_{1}, x_{2}, \cdots, x_{n} ; \epsilon\right)$, then for any $C^{\prime} \in \Re^{\prime}$ there exists an $A^{\prime} \in \Re^{\prime}$ such that $\left\|C^{\prime}\left(A-A^{\prime}\right) x_{i}\right\|<\epsilon$ and $\left\|\left(A-A^{\prime}\right) C^{\prime} x_{i}\right\|<\epsilon$ for $i=1,2, \cdots, n$. Hence $A C^{\prime}=C^{\prime} A$ belongs to $\Re$. Likewise there exists a $B^{\prime} \in \Re^{\prime}$ such that $\left\|A\left(B-B^{\prime}\right) x_{i}\right\|<\epsilon$ and $\left\|\left(B-B^{\prime}\right) A x_{i}\right\|<\epsilon$ for $i=1, \cdots, n$. Since as above $A B^{\prime}=B^{\prime} A \in \Re$, there exists a $D^{\prime} \in \Re^{\prime}$ such that $\left\|\left(A B^{\prime}-D^{\prime}\right) x_{i}\right\|$ $<\epsilon$. Combining these inequalities we get $\left\|\left(A B-D^{\prime}\right) x_{i}\right\|<2 \epsilon$ and $\left\|\left(B A-D^{\prime}\right) x_{i}\right\|$ $<2 \epsilon$. Hence $A B=B A$ belongs to $\Re$.

We now adjoin to $\Re_{1}$ all inverses in $\mathfrak{E}(\mathfrak{X})$ of elements in $\Re_{1}$. The resulting set $\mathfrak{A}$ is clearly abelian. Finally we define $\Re$ to be the strong closure of all polynomials in $\mathfrak{A}$. As before $\mathfrak{R}$ will be a commutative Banach algebra with unit $\left({ }^{3}\right)$.

Since $\mathfrak{R}_{1}$ is the strong closure of polynomials in $\mathfrak{A}_{1}$, it will in particular contain the elements $\Theta(\alpha)$ defined by

$$
\Theta(\alpha) x=\int_{0}^{\infty} T(t) x d \alpha
$$

for $\alpha \in S(\omega)$. Further it is clear that

$$
\begin{aligned}
\Theta\left(e_{0}\right) & =I \\
\Theta(a \alpha+b \beta) & =a \Theta(\alpha)+b \Theta(\beta), \\
\Theta(\alpha \beta) x & =\int_{0}^{\infty} T(t) x d \gamma=\int_{0}^{\infty} \int_{0}^{\infty} T(u) T(v) x d_{v} \beta d_{u} \alpha \\
& =\int_{0}^{\infty} T(u) \Theta(\beta) x d_{u} \alpha=\Theta(\alpha)[\Theta(\beta) x] \\
\|\Theta(\alpha)\| & \leqq \int_{0}^{\infty}\|T(t)\||d \alpha| \leqq\|\alpha\| .
\end{aligned}
$$

Hence (13) defines a continuous homeomorphism of $\subseteq(\omega)$ into $\Re$ which takes the unit $e_{0}$ into the identity $I$. The mapping $\Theta(\alpha)$ can be thought of as defining an operational calculus for the infinitesimal generator $A$ (see [6, chap. 15]). It should also be pointed out that for $x \in \mathfrak{D}(A), A \Theta(\alpha) x=\Theta(\alpha) A x$. This follows from the fact that $A$ is closed and that $\Theta(\alpha) x=\int_{0}^{\infty} T(t) x d \alpha$ is the limit of partial sums each of which lie in $\mathfrak{D}(A)$.

We next apply the Gelfand theory [3] to the commutative Banach algebra with unit, $\Re$. Let $\mathfrak{M}^{\prime}$ be the set of all maximal ideals in $\Re$, topologized in the usual way to form a compact Hausdorff space. As $m^{\prime}$ ranges over $\mathfrak{M}^{\prime}$, the function $B\left(m^{\prime}\right)=\mu_{m^{\prime}}(B)$ is continuous on $\mathfrak{M}^{\prime}$ for each $B \in \Re$. The range of $B\left(m^{\prime}\right)$ consists precisely of the spectrum of $B$ relative to $\Re\left({ }^{4}\right)$. For

$\left.{ }^{3}\right)$ Any commutative Banach algebra contained in $\mathfrak{E}(\mathfrak{X})$ and containing $\Re$ could be used instead of $\Re$.

(4) The symbol $\Sigma(B)$ is used exclusively for the spectrum of $B$ relative to $(\mathfrak{E}(\mathfrak{X})$. 
each $B \in \Re_{1}, \Re$ includes $R[\lambda ; B]$ for $\lambda \in \rho(B)$; hence the range of $B\left(m^{\prime}\right)$ is likewise the spectrum of $B \in \Re_{1}$ relative to $\mathfrak{E}(\mathfrak{X})$. One of the important results of the Gelfand theory is that the spectral radius

$$
\|\mid B\|=\sup \left[\left|B\left(m^{\prime}\right)\right| \mid m^{\prime} \in \mathfrak{M}^{\prime}\right]=\lim _{n \rightarrow 0}\left\|B^{n}\right\|{ }^{1 / n} .
$$

Given a maximal ideal $m^{\prime} \in \mathfrak{M}^{\prime}$ and its associated multiplicative linear functional $\mu^{\prime}$, then

$$
\mu^{\prime}[\Theta(\alpha)]=\mu(\alpha)
$$

clearly defines a multiplicative linear functional $\mu$ on $\subseteq(\omega)$. Since $\Theta\left(e_{0}\right)=I, \mu$ is nondegenerate. Thus $\Theta$ induces a mapping $\Phi$ on $\mathfrak{M}^{\prime}$ into $\mathfrak{M}$ such that

$$
\Theta(\alpha)\left(m^{\prime}\right)=\alpha\left[\Phi\left(m^{\prime}\right)\right] .
$$

It is easy to see that $\Phi$ is a continuous mapping. For, given a neighborhood $\mathfrak{B}=\left[m|| \alpha_{i}(m)-\alpha_{i}\left(m_{0}\right) \mid<\epsilon ; i=1, \cdots, n\right]$ of $m_{0}=\Phi\left(m_{0}^{\prime}\right)$, then the $\Phi$ image of $\mathfrak{B}^{\prime}=\left[m^{\prime}|| \Theta\left(\alpha_{i}\right)\left(m^{\prime}\right)-\Theta\left(\alpha_{i}\right)\left(m_{0}^{\prime}\right) \mid<\epsilon ; i=1,2, \cdots, n\right]$ is contained in $\mathfrak{B}$.

Corresponding to the sets $\mathfrak{B}, \mathfrak{Z}, \mathfrak{U}$ defined by (8), we now define

$$
\mathfrak{B}^{\prime}=\Phi^{-1}(\mathfrak{B}), \quad \mathbb{Z}^{\prime}=\Phi^{-1}(\mathfrak{Z}), \quad \mathfrak{u}^{\prime}=\Phi^{-1}(\mathfrak{U}) .
$$

It follows from section two that the sets $\mathfrak{B}^{\prime}, \mathfrak{Z}^{\prime}, \mathfrak{u}^{\prime}$ form a mutually disjoint subdivision of $\mathfrak{M}^{\prime}$. Since $\mathfrak{B}$ is an open subset of $\mathfrak{M}$ and since $\Phi$ is continuous, $\mathfrak{W}^{\prime}$ will likewise be an open subset of $\mathfrak{M}^{\prime}$. We note that $\Theta\left(e_{t}\right)=T(t)$ and hence that $T(t)\left(m^{\prime}\right)=e_{t}\left[\Phi\left(m^{\prime}\right)\right]$. Making use of the theory developed in section two it follows that (a) if $m^{\prime} \in \mathfrak{B}^{\prime}$, then there exists a complex number $a\left(m^{\prime}\right)$ with $R\left[a\left(m^{\prime}\right)\right] \leqq \omega_{0}$ such that $T(t)\left(m^{\prime}\right)=\exp \left[a\left(m^{\prime}\right) t\right]$; (b) if $m^{\prime} \in Z^{\prime}$, then $T(t)\left(m^{\prime}\right)=1$ for $t=0$ and zero for $t>0$ and hence $0 \in \Sigma(T(t))$ for $t>0$; and finally (c) if $m^{\prime} \in \mathfrak{U}^{\prime}, T(t)\left(m^{\prime}\right)=\chi(t) \cdot \exp (\xi t)$ where $\xi \leqq \omega_{0}$ and $\chi(t)$ is a character of the real line. Moreover if $\alpha \in \mathfrak{R}(\omega)$, then

$$
\begin{aligned}
\Theta(\alpha)\left(m^{\prime}\right) & =\int_{0}^{\infty} \exp \left[a\left(m^{\prime}\right) t\right] d \alpha & & \left(m^{\prime} \in \mathfrak{W}^{\prime}\right) \\
& =0 & & \text { elsewhere. }
\end{aligned}
$$

In particular if $d \alpha([0, t]) / d t=\exp (-\lambda t)$ with $R(\lambda)>\omega_{0}$, then by (12)

$$
\begin{aligned}
R(\lambda ; A)\left(m^{\prime}\right) & =\left[\lambda-a\left(m^{\prime}\right)\right]^{-1} & & \left(m^{\prime} \in \mathfrak{B}^{\prime}\right) \\
& =0 & & \text { elsewhere. }
\end{aligned}
$$

THEOREM 3.1. The relation (18) is valid for all $\lambda \in \rho(A)$ and $\Sigma(A)$ $=\left[a\left(m^{\prime}\right) \mid m^{\prime} \in \mathfrak{W}^{\prime}\right]$. Further $\mathbb{R}[\Sigma(A)] \leqq \omega_{0}$.

Suppose now that $\lambda, \zeta \in \rho(A)$, then the first resolvent equation (10) is satisfied. Hence for $R(\lambda)>\omega_{0}$, 


$$
\begin{array}{cr}
R(\zeta ; A)\left(m^{\prime}\right)-\left[\lambda-a\left(m^{\prime}\right)\right]^{-1}=(\lambda-\zeta)\left[\lambda-a\left(m^{\prime}\right)\right]^{-1} R(\zeta ; A)\left(m^{\prime}\right) & \text { on } \mathfrak{W}^{\prime}, \\
R(\zeta ; A)\left(m^{\prime}\right)-0=0 \cdot R(\zeta ; A)\left(m^{\prime}\right) & \text { elsewhere, }
\end{array}
$$

from which it follows that (18) is valid with $\lambda$ replaced by $\zeta$. In particular, $a\left(m^{\prime}\right)$ must belong to the set $\Sigma(A)$. Conversely suppose that $\zeta$ is not in the range of $\left[a\left(m^{\prime}\right) \mid m^{\prime} \in \mathfrak{B}^{\prime}\right]$ and that $R(\lambda)>\omega_{0}$, then

$$
\begin{array}{rlr}
{[I+(\zeta-\lambda) R(\lambda ; A)]\left(m^{\prime}\right)} & =\left[\zeta-a\left(m^{\prime}\right)\right]\left[\lambda-a\left(m^{\prime}\right)\right]^{-1} & \text { on } \mathfrak{W}^{\prime} \\
& =1 & \text { elsewhere. }
\end{array}
$$

Since $[I+(\zeta-\lambda) R(\lambda ; A)]\left(m^{\prime}\right) \neq 0$ on $\mathfrak{M}^{\prime}$, the inverse of $[I+(\zeta-\lambda) R(\lambda ; A)]$ exists in $\Re$. If we set

$$
R(\zeta)=R(\lambda ; A)[I+(\zeta-\lambda) R(\lambda ; A)]^{-1},
$$

then for all $x \in \mathfrak{X}$

$$
\begin{aligned}
(\zeta I-A) R(\zeta) x & =[(\zeta-\lambda) I+(\lambda I-A)] R(\lambda ; A)[I+(\zeta-\lambda) R(\lambda ; A)]^{-1} x \\
& =[(\zeta-\lambda) R(\lambda ; A)+I][I+(\zeta-\lambda) R(\lambda ; A)]^{-1} x=x,
\end{aligned}
$$

whereas for $x \in \mathfrak{D}(A)$

$$
\begin{aligned}
R(\zeta)(\zeta I-A) x & =[I+(\zeta-\lambda) R(\lambda ; A)]^{-1} R(\lambda ; A)[(\zeta-\lambda) I+(\lambda I-A)] x \\
& =[I+(\zeta-\lambda) R(\lambda ; A)]^{-1}[(\zeta-\lambda) R(\lambda ; A)+I] x=x .
\end{aligned}
$$

Hence $R(\zeta)$ is actually the resolvent of $A$ at $\zeta$ and $\zeta \in \rho(A)$.

This concludes our preliminary remarks. We now go on to a detailed study of various types of semi-groups of operators.

4. $T(t)$ uniformly continuous at $t=0$. If $T(t)$ is continuous in the uniform topology at $t=0$, then its infinitesimal generator $A$ is a bounded linear transformation and conversely (see [6, chap. 8]). In fact $A=\lim _{\tau \rightarrow 0} \tau^{-1}[T(\tau)-I]$ exists in the uniform topology and $T(t)=\exp (A t)$. Hence $A$ belongs to the ring $\Re$ introduced in section three and has the representation $A\left(m^{\prime}\right)=a\left(m^{\prime}\right)$ as a continuous function over the maximal ideals $\mathfrak{M}^{\prime}$ of $\mathfrak{R}$. Likewise $T(t)\left(m^{\prime}\right)$ $=\exp \left[a\left(m^{\prime}\right) t\right]$.

THEOREM 4.1. For a semi-group of operators $T(t)$ of type $(\mathrm{H})$, the following statements are equivalent:

(a) $T(t)=\exp (A t)$ where $A$ is a bounded linear transformation.

(b) $\mathfrak{M}^{\prime}=\mathfrak{B}^{\prime}$.

(c) $T(t)$ has the representation $T(t)\left(m^{\prime}\right)=\exp \left[a\left(m^{\prime}\right) t\right]$ over the maximal ideals $\mathfrak{M}^{\prime}$ of $\Re$.

Suppose $T(t)=\exp (A t)$ where $A \in \mathfrak{E}(\mathfrak{X})$. Then $T(t)$ is continuous in the uniform topology for $t \geqq 0$, and hence $\int_{0}^{\tau} T(t) d t$ exists in the uniform topology. Now the multiplicative linear functionals $\mu^{\prime}$ are continuous in the uniform topology so that 


$$
\begin{aligned}
\mu^{\prime}\left[\int_{0}^{\tau} T(t) d t\right] & =\int_{0}^{\tau} \mu^{\prime}[T(t)] d t=\int_{0}^{\tau} \exp \left[\mu^{\prime}(A) t\right] d t \\
& =\tau \text { or }\left\{\exp \left[\mu^{\prime}(A) \tau\right]-1\right\} / \mu^{\prime}(A)
\end{aligned}
$$

according as $\mu^{\prime}(A)$ is or is not zero. Now $\alpha_{0}([0, t])=t$ for $0 \leqq t \leqq \tau$ and $=\tau$ for $t \geqq \tau$ belongs to $\mathfrak{R}(\omega)$ and

$$
\mu^{\prime}\left[\int_{0}^{\tau} T(t) d t\right]=\mu^{\prime}\left[\Theta\left(\alpha_{0}\right)\right]=\Theta\left(\alpha_{0}\right)\left(m^{\prime}\right)=\alpha_{0}\left[\Phi\left(m^{\prime}\right)\right] \neq 0
$$

for $\tau$ sufficiently small. Hence $m^{\prime} \in \mathfrak{W}^{\prime}$ for all $m^{\prime} \in \mathfrak{M}^{\prime}$. Thus (a) implies (b). By Theorem 2.1, if $m^{\prime} \in \mathfrak{W}^{\prime}$ there exists a complex number $a\left(m^{\prime}\right)$ such that $T(t)\left(m^{\prime}\right)=e_{t}\left[\Phi\left(m^{\prime}\right)\right]=\exp \left[a\left(m^{\prime}\right) t\right]$. Hence (b) implies (c).

It remains to prove that (c) implies (a). Here we assume merely that the representation of $T(t)$ is of the form $\exp \left[a\left(m^{\prime}\right) t\right]$ and that this function is continuous in $m^{\prime}$ for each $t \geqq 0$. We first show that $a\left(m^{\prime}\right)$ is itself continuous on $\mathfrak{M}^{\prime}$ and later that there exists a bounded linear transformation $A$ such that $T(t)=\exp (A t)$.

Lemma 4.1. If $g_{t}\left(m^{\prime}\right)=\exp \left[a\left(m^{\prime}\right) t\right]$ is continuous for each $t \geqq 0$ in the compact Hausdorff space $\mathfrak{M}^{\prime}$, then $a\left(m^{\prime}\right)$ is itself continuous on $\mathfrak{M}^{\prime}$.

Since $g_{1}\left(m^{\prime}\right) \neq 0$ and is continuous on $\mathfrak{M}^{\prime}, 0<c \leqq\left|g_{1}\left(m^{\prime}\right)\right| \leqq k$. Hence $R\left[a\left(m^{\prime}\right)\right]$ is bounded. Suppose that $\left|J\left[a\left(m^{\prime}\right)\right]\right|$ were not bounded. Then we can find a sequence $\left\{m_{n}^{\prime}\right\}$ such that $R\left[a\left(m_{n}^{\prime}\right)\right] \rightarrow b$ and $J\left[a\left(m_{n}^{\prime}\right)\right] \rightarrow \infty$ (or $-\infty$ ). Since $\left|g_{t}\left(m^{\prime}\right)\right|$ is bounded on $\mathfrak{M}^{\prime}$, we can then find a subsequence (which we renumber) such that $g_{t}\left(m_{n}^{\prime}\right)$ converges to a limit as $n \rightarrow \infty$ for each $t$ of a denumerable dense subset $\mathfrak{B}$ of $[0, \infty)$. Since $\mathfrak{M}^{\prime}$ is compact there exists at least one point of condensation $m_{0}^{\prime}$ of the set $\left\{m_{n}^{\prime}\right\}$. Now $a\left(m_{0}^{\prime}\right)$ will be of the form $(b+i d)$. Further since $g_{t}\left(m^{\prime}\right)$ is continuous in each variable, it follows that

$$
h_{t}\left(m_{n}^{\prime}\right) \equiv \exp \left[\left\{a\left(m_{n}^{\prime}\right)-(b+i d)\right\} t\right] \rightarrow 1
$$

as $n \rightarrow \infty$

for $t \in \mathfrak{B}$. We now define

$$
\mathfrak{Q}_{n}=\left[t \mid 0 \leqq t \leqq 2 \pi ; \pi / 2 \leqq \arg h_{t}\left(m_{n}^{\prime}\right) \leqq 3 \pi / 2\right] .
$$

Because of the fact that $\Im\left[a\left(m_{n}^{\prime}\right)-(b+i d)\right] \rightarrow \infty$ with $n$, we have, for sufficiently large $n$, meas $\left(\mathfrak{Q}_{n}\right) \geqq 1$. Hence meas $\left(\lim\right.$ sup $\left.\mathfrak{Q}_{n}\right) \geqq 1$. For any $t_{0}$ $\in \lim \sup \mathfrak{Q}_{n}$, we choose a subsequence of the $n$ 's (again we renumber) such that $h_{t_{0}}\left(m_{n}^{\prime}\right) \rightarrow \exp \left(i \phi_{0}\right)$ with $\pi / 2 \leqq \phi_{0} \leqq 3 \pi / 2$. We now have

$$
\begin{aligned}
g_{t}\left(m_{n}^{\prime}\right) & \rightarrow \exp [(b+i d) t], & t \in \mathfrak{P}, \\
g_{t_{0}}\left(m_{n}^{\prime}\right) & \rightarrow \exp \left(i \phi_{0}\right) \exp \left[(b+i d) t_{0}\right] . &
\end{aligned}
$$

For no condensation point $m^{\prime}$ of this final subsequence can $g_{t}\left(m^{\prime}\right)$ be of the 
form $\exp \left[a\left(m^{\prime}\right) t\right]$ and hence continuous in $t$ since it must be equal to the right sides of (19) both on the dense set $\mathfrak{B}$ and on $t_{0}$. It therefore follows that $\left|\mathfrak{J}\left[a\left(m^{\prime}\right)\right]\right|$ is bounded on $\mathfrak{M}^{\prime}$. This implies that $\left(g_{\tau}\left(m^{\prime}\right)-1\right) / \tau$ converges uniformly on $\mathfrak{M}^{\prime}$ as $\tau \rightarrow 0$. Thus $a\left(m^{\prime}\right)$ is the uniform limit of continuous functions and is therefore itself continuous on $\mathfrak{M}^{\prime}$.

We now return to the proof of Theorem 4.1. By our lemma $\left|a\left(m^{\prime}\right)\right|$ is bounded, say by $k$. Hence for $t<\pi / 2 k$, exp $\left[a\left(m^{\prime}\right) t\right]$ lies in the interior of the right half-plane for all $m^{\prime} \in \mathfrak{M}^{\prime}$. Hence $t^{-1} \log [T(t)] \equiv A_{t}$ is a well defined element of $\Re$ (see Hille [6, chap. 5]), and $A_{t}\left(m^{\prime}\right)=a\left(m^{\prime}\right)$. If $\Re$ had no radical, then we could assert that $A_{t}$ is independent of $t$ and hence that $T(t)=\exp (A t)$. Allowing for the possibility of a radical we argue as follows. For notational convenience suppose that $2 k<\pi$ and set $A_{1}=A$. It is clear that $T(n)$ $=\exp (A n)$ and therefore that the semi-group

$$
S(t)=\exp (-A t) T(t)
$$

is periodic of period one. Since $T(t)$ satisfies the hypothesis $(\mathrm{H})$ and since $\exp (-A t)$ is continuous in the uniform topology, it follows that $S(t)$ also satisfies the hypothesis $(\mathrm{H})$. Now $S(t)$, being of period one, must be strongly continuous everywhere and uniformly bounded in norm and this remains true even if we extend $S(t)$ periodically over $(-\infty, \infty)$. Finally the representation of $S(t)$ is given by $S(t)\left(m^{\prime}\right)=\exp \left[-a\left(m^{\prime}\right) t\right] \exp \left[a\left(m^{\prime}\right)\right] t=1$ and hence $S(t)$ differs from the identity at most by a generalized nilpotent element of $\Re$. It now follows from a rather deep theorem due to Gelfand [4] (see also $[6$, p. 493] and [15]) that $S(t)=I$ and hence that $T(t)=\exp (A t)$. For the sake of completeness we shall give a simpler proof of this fact which applies to our special situation. We define

$$
J_{n}(x)=\int_{0}^{1} S(t) x \exp (-2 \pi i n t) d t .
$$

It can be shown by direct computation that $J_{n} J_{m}=\delta_{m n} J_{n}$; that is, the $J_{n}$ 's form an orthogonal system of projection operators. Further $J_{n} S(t) x$ $=J_{n}(x) \exp (2 \pi i n t)$. By the analogue of the classical Fejér Theorem on Fourier series, $S(t)=(C-1) \sum_{-\infty}^{\infty} J_{n}(x) \exp (2 \pi i n t)$ and in particular $S(0) x$ $=x=(C-1) \sum_{-\infty}^{\infty} J_{n}(x)$. Since $J_{n}$ is idempotent, $J_{n}\left(m^{\prime}\right)=0$ or 1 and since $\left\|\left|J_{n}\left\|\mid=\lim _{k \rightarrow \infty}\right\| J_{n}^{k}\left\|^{1 / k}=\lim _{k \rightarrow \infty}\right\| J_{n} \| 1 / k\right.\right.$, it follows that $J_{n}\left(m^{\prime}\right) \equiv 0$ if and only if $J_{n}=0$. Hence

$J_{n}\left(m^{\prime}\right)=J_{n}\left(m^{\prime}\right) \cdot S(t)\left(m^{\prime}\right)=\left[J_{n} S(t)\right]\left(m^{\prime}\right)=J_{n}\left(m^{\prime}\right) \exp (2 \pi i n t) \quad$ for all $t$

implies that $J_{n}\left(m^{\prime}\right) \equiv 0$ and therefore that $J_{n}=0$ for $n \neq 0$. Thus $J_{0}=S(0)=I$ and $S(t) \equiv I$.

COROLlaRY 4.1. If $T(t)$ is a semi-group of operators of type $(\mathrm{H})$ and if there exists an unbounded open connected set \& containing the origin such that for 
some interval $0 \leqq a<t<b, \Sigma[T(t)] \cap(5)=\phi$, then the infinitesimal generator $A$ is bounded and $T(t)=\exp (A t)$.

Since zero does not belong to $\Sigma[T(t)]$ for some $t_{0}>0, T\left(t_{0}\right)\left(m^{\prime}\right) \neq 0$ and therefore $\mathfrak{Z}^{\prime}$ is vacuous. If for some $m_{0}^{\prime}, T(t)\left(m_{0}^{\prime}\right)=\exp (\xi t) \chi(t)$ where $\chi(t)$ is a nonmeasurable character of the real line, then the values of arg $\left[T(t)\left(m_{0}\right)\right]$ would, by Lemma 2.1 , be dense on the unit circle in every interval $0 \leqq a<t<b$ and this is easily seen to contradict our hypothesis on $\$(5$. Hence we conclude that $T(t)\left(m^{\prime}\right)$ must be of the form exp $\left[a\left(m^{\prime}\right) t\right]$ and the result now follows directly from Theorem 4.1. This corollary generalizes a theorem due to Hille [6, Theorem 13.7.1] by relaxing conditions on $(\$)$, on the interval $(a, b)$, and on the semi-group of operators $T(t)$.

Corollary 4.2. If $T(t)$ is a semi-group of operators of type $(\mathrm{H})$, then the following statements are equivalent:

(a) The infinitesimal generator $A$ of $T(t)$ is unbounded.

(b) $\mathfrak{W}^{\prime}$ is a proper subset of $\mathfrak{M}^{\prime}$.

(c) $0 \in \Sigma[R(\lambda ; A)]$ for all $\lambda$ of $\rho(A)\left({ }^{5}\right)$.

If we now define for $\alpha \in \mathfrak{S}(\omega)$ the function

$$
f_{\alpha}(\lambda)=\int_{0}^{\infty} \exp (\lambda t) d \alpha
$$

for $R(\lambda) \leqq \omega_{0}$, then it is clear by Theorem 3.1 that $f_{\alpha}[\Sigma(A)]$ is precisely the range of values of $\Theta(\alpha)\left(m^{\prime}\right)$ on $\mathfrak{W}^{\prime}$. Thus we obtain the following theorem.

THEOREM 4.2. If the semi-group of operators $T(t)$ is uniformly continuous at $t=0$, then $\Sigma[\Theta(\alpha)]=f_{\alpha}[\Sigma(A)]$ for all $\alpha \in \Im(\omega)$. If the semi-group of operators $T(t)$ of type $(\mathrm{H})$ has an unbounded infinitesimal generator $A$, then $\Sigma[\Theta(\alpha)]$ $\supset f_{\alpha}[\Sigma(A)]$ for all $\alpha \in \Im(\omega)$ whereas for $\alpha \in \mathbb{R}(\omega), \Sigma[\Theta(\alpha)]=f_{\alpha}[\Sigma(A)] \cup 0$.

It is clear that for $\alpha \in \mathfrak{R}(\omega), \Theta(\alpha)\left(m^{\prime}\right)=0$ on $\mathfrak{M}^{\prime}-\mathfrak{W}^{\prime}$ and hence that $0 \in \Sigma[\Theta(\alpha)]$ when $A$ is unbounded( $\left(^{5}\right)$. Theorem 4.2 in essentially $\left(^{6}\right)$ this form was first proved by Hille [6, Theorem 15.5.1].

It is worth remarking that if for some $\alpha \in \mathbb{R}(\omega),[\Theta(\alpha)]^{-1}$ exists, then $\mathfrak{W}^{\prime}=\mathfrak{M}^{\prime}$ and hence $T(t)=\exp (A t)$ where $A$ is a bounded linear operator. On the other hand if for some $\alpha \in \Im(\omega)$ with $\alpha(0)=0,[\Theta(\alpha)]^{-1}$ exists, then $乃^{\prime}$ must be vacuous and therefore $T(t)^{-1}$ exists. In this case $T(t)$ can be embedded in a strongly continuous group of operators defined on $(-\infty, \infty)$.

5. $T(t)$ uniformly continuous for $t \geqq c>0$. In this section we shall deal with semi-groups of operators $T(t)$ of type $(\mathrm{H})$ which are continuous in the

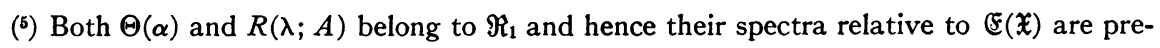
cisely the ranges of $\Theta(\alpha)\left(m^{\prime}\right)$ and $R(\lambda ; A)\left(m^{\prime}\right)$ respectively.

$\left({ }^{(}\right)$There is an unresolved difficulty about 0 belonging to $\Sigma[\Theta(\alpha)]$ in Hille's proof for the case $\alpha \in \mathfrak{R}(\omega)$ and $A$ unbounded. 
uniform topology for $t \geqq c>0\left({ }^{7}\right)$. We shall explicitly assume that $T(t)$ is not continuous in the uniform topology at $t=0$ and hence that the infinitesimal generator $A$ is unbounded. For any linear multiplicative functional $\mu^{\prime}$ on $\Re$, $\mu^{\prime}(T(t))=\mu^{\prime}\left[\Theta\left(e_{t}\right)\right]=\mu\left(e_{t}\right)$ will be continuous for $t \geqq c>0$. This is enough to exclude nonmeasurable characters. However more can be asserted; for clearly

$$
\mu^{\prime}\left[\tau^{-1} \int_{c}^{c+\tau} T(t) d t\right]=\tau^{-1} \int_{c}^{c+r} \mu^{\prime}[T(t)] d t \rightarrow \mu^{\prime}[T(c)]
$$

as $\tau \rightarrow 0$. But $\tau^{-1} \int_{c}^{c+\tau} T(t) d t=\Theta(\alpha)$ where $\alpha([0, t])=0$ for $t<c,=(t-c) / \tau$ for $c \leqq t \leqq c+\tau,=1$ for $t>c+\tau$, and $\alpha$ belongs to $\mathfrak{l}(\omega)$. Hence if $\mu^{\prime}[T(c)]=0$ the corresponding $m^{\prime} \in \mathfrak{Z}^{\prime}$ and otherwise $m^{\prime} \in \mathfrak{B}^{\prime}$. Since we have assumed the infinitesimal generator $A$ to be unbounded, $\mathfrak{Z}^{\prime}=\mathfrak{M}^{\prime}-\mathfrak{W}^{\prime}$ will not be empty (Corollary 4.2). It now follows from Theorems 2.1 and 2.2 that

$$
\Sigma[\Theta(\alpha)]=f_{\alpha}[\Sigma(A)] \cup \alpha(0)
$$

for all $\alpha \in \subseteq(\omega)$. We shall even be able to obtain a correspondence between the detailed spectrum of $\Theta(\alpha)$ and $A$. To this end we prove the following lemma.

LEMMA 5.1. If the semi-group of operators $T(t)$ is of type $(\mathrm{H})$ and continuous in the uniform topology for $t \geqq c>0$, then $\Sigma(A)$ lies to the left of a bounding curve $\xi=\psi(|\eta|)(\lambda=\xi+i \eta)$ where $\lim _{\eta \rightarrow \infty} \psi(\eta)=-\infty$.

By Theorem 3.1, $\Sigma(A)=\left[a\left(m^{\prime}\right) \mid m^{\prime} \in \mathfrak{B}^{\prime}\right]$. Hence if the lemma were false, there would exist a subsequence $\left\{m_{n}^{\prime}\right\} \subset \mathfrak{W}^{\prime}$ such that $R\left[a\left(m_{n}^{\prime}\right)\right] \rightarrow \xi_{0} \leqq \omega_{0}$ and $\Im\left[a\left(m_{n}^{\prime}\right)\right] \rightarrow \infty($ or $-\infty)$. By the Riemann-Lebesgue Theorem, we have

$$
\lim _{n \rightarrow \infty} \int_{0}^{\infty} \exp \left[a\left(m_{n}^{\prime}\right) t\right] d \alpha=0
$$

for all $\alpha \in \mathfrak{R}(\omega)$. However, since $\mathfrak{M}^{\prime}$ is compact, $\left\{m_{n}^{\prime}\right\}$ has at least one point of condensation, say $m_{0}^{\prime}$. It follows from (22) that $\Theta(\alpha)\left(m_{0}^{\prime}\right)=0$ for all $\alpha \in \mathcal{R}(\omega)$ and hence that $m_{0}^{\prime} \notin \mathfrak{W}^{\prime}$. On the other hand, $\left|\Theta\left(e_{t}\right)\left(m_{n}^{\prime}\right)\right| \rightarrow \exp \left(\xi_{0} t\right)$ and hence $m_{0}^{\prime} \notin \mathfrak{Z}^{\prime}$. This is contrary to the fact that $\mathfrak{M}^{\prime}=\mathfrak{W}^{\prime} \cup \mathfrak{Z}^{\prime}$.

If we impose further restrictions on the semi-group of operators, the function $\psi(\eta)$ can be further delimited. Hille [7] has considered semi-group operators (strongly continuous for $t>0$ ) which map $\mathfrak{X}$ into $\mathfrak{D}(A)$. In this case $d^{n} T(t) x /(d t)^{n}=A^{n} T(t) x$ defines a bounded operator on $X$ for $t>0[7$, Theorem $1]$.

ThEOREM 5.1. Let $T(t)$ be a semi-group of operators of type $(\mathrm{H})$ such that $T(t)$ maps $\mathfrak{X}$ into $\mathfrak{D}(A)$ if $t>0$. Then $\Sigma(A)$ lies to the left of a bounding curve $\xi=K-\log |\eta|(\lambda=\xi+i \eta)$.

( ${ }^{7}$ The assumption that $T(t)$ is continuous in the uniform topology for $t \geqq c>0$ is equivalent to $T(t)$ being weakly measurable and $[T(t) \mid t \geqq c>0]$ being a separable subset of $\mathbb{E}(\mathfrak{X})$. This follows from Theorems 3.3.1 and 8.3.1 of Hille [6]. 
It is readily seen that $T(t)$ will be continuous in the uniform topology for $t>0$. For

$$
T(b)-T(a)=\int_{a}^{b} A T(t) d t, \quad 0<a<b,
$$

and since $A T(a)$ is a bounded transformation,

$$
\|T(b)-T(a)\| \leqq\|A T(a)\| \int_{0}^{b-a}\|T(t)\| d t .
$$

The right side converges to zero as $b \rightarrow a$ uniformly for $1 / \delta<a<\delta<\infty$. More is true; namely, the derivative exists in the uniform topology. This is seen from the equation

$$
[T(a+\Delta)-T(a)] / \Delta-A T(a)=\Delta^{-1} \int_{a}^{a+\Delta}[A T(t)-A T(a)] d t \quad(a, \Delta>0)
$$

from which it follows that

$$
\|[T(a+\Delta)-T(a)] / \Delta-A T(a)\| \leqq\|A T(a / 2)\| \int_{0}^{\Delta}\|T(t-a / 2)-T(a / 2)\| d t .
$$

Since $T(t)$ is continuous in the uniform topology for $t>0$, the right-hand side of this inequality goes to zero with $\Delta$. A similar argument holds for $\Delta<0$. Making use of the above representation argument, we see that $T(t)\left(m^{\prime}\right)$ $=\exp \left[a\left(m^{\prime}\right) t\right]$ on $\mathfrak{B}^{\prime}$ and 0 on $\mathfrak{Z}^{\prime}$ for $t>0$. Since the derivative exists in the uniform topology, we see that $(A T)\left(m^{\prime}\right)=a\left(m^{\prime}\right) \exp \left[a\left(m^{\prime}\right) t\right]$ in $\mathfrak{B}^{\prime}$ and 0 on $Z^{\prime}$ for $t>0$. Hence

$$
\text { LUB }\left[\left|a\left(m^{\prime}\right)\right| \exp \left[a\left(m^{\prime}\right) t\right] \mid m^{\prime} \in \mathfrak{W}^{\prime}\right] \leqq K(t) \equiv\|A T(t)\| .
$$

In other words, $\Sigma(A)$ lies to the left of the curve $\left(\xi^{2}+\eta^{2}\right)^{1 / 2} \exp [\xi t]=K(t)$ and a fortiori to the left of $|\eta| \exp [\xi t]=K(t)$, which can be written as $\xi=K-\log |\eta|$ when $t=1$.

Definition. The operator $T$ has the property (see Hille [6, p. 313]):

$\mathrm{P}_{1}$ if there is an $x_{0} \neq 0$ such that $T x_{0}=0$;

$\mathrm{P}_{2}$ if the range of $T$ is not dense in $X$.

$\mathrm{P}_{3}$ if the $T$-image of the intersection of the domain of $T$ with the shell of the unit sphere is not bounded away from the zero element.

ThEOREM 5.2. Let $T(t)$ be a semi-group of operators of type $(\mathrm{H})$ continuous in the uniform topology for $t \geqq c>0$ and let $\alpha \in \Im\left(\omega^{\prime}\right)$ where $\omega^{\prime}(t)=\max [\omega(t)$, $\left.\omega_{1} t\right]$ for some $\omega_{1}>\omega_{0}\left({ }^{8}\right)$. If $\nu \in \Sigma(A)$ and if $[\nu I-A]$ has property $\mathrm{P}_{j}$, then $\left[f_{\alpha}(\nu) I\right.$

(8) The maximum of two subadditive functions is again a subadditive function. 
$-\Theta(\alpha)]$ has the same property $\mathrm{P}_{j}$. Conversely $[\mu I-\Theta(\alpha)], \mu \neq \alpha(0)$, has property $\mathrm{P}_{j}$ only if there is a $\nu \in \Sigma(A)$ with $f_{\alpha}(\nu)=\mu$ such that $[\nu I-A]$ has the property $\mathrm{P}_{j}$. There is one exception: if $[\mu I-\Theta(\alpha)]$ has $\mathrm{P}_{3}$ together with $\mathrm{P}_{1}$ or $\mathrm{P}_{2}$, then $\mathrm{P}_{3}$ may possibly not hold for any $[\nu I-A]$, where $f_{\alpha}(\nu)=\mu$ and $\nu \in \Sigma(A)$.

The proof of this theorem makes use of a method due to Hille [6, Theorem 15.5.2]. Suppose first that $\nu \in \Sigma(A)$ and that $\alpha \in \subseteq\left(\omega^{\prime}\right)$. Then

$$
f_{\beta}(\lambda)=\left[f_{\alpha}(\nu)-f_{\alpha}(\lambda)\right] /(\nu-\lambda)=\int_{0}^{\infty} \exp (\lambda t) d_{t} \beta
$$

where

$$
\beta^{\prime}(t)=\int_{0}^{t} \exp [-\nu(t-s)] d_{s}\left[f_{\alpha}(\nu) e_{0}-\alpha\right]=\int_{t}^{\infty} \exp [\nu(s-t)] d_{s} \alpha .
$$

It is easily seen that $\beta \in S(\omega)$. For by Theorem $3.1, R(\nu) \leqq \omega_{0}$ and hence

$$
\begin{aligned}
\|\beta\| & \leqq \int_{0}^{\infty} \exp [\omega(t)] \int_{t}^{\infty} \exp \left[\omega_{1}(s-t)\right]\left|d_{s} \alpha\right| d t \\
& \leqq \int_{0}^{\infty} \exp \left[\omega(t)-\omega_{1} t\right] d t \int_{0}^{\infty} \exp \left(\omega_{1} s\right)\left|d_{s} \alpha\right|<\infty .
\end{aligned}
$$

Thus $\Theta(\beta)$ exists. We should like to show that

$$
\begin{aligned}
f_{\alpha}(\nu) I-\Theta(\alpha) & =[\nu I-A] \Theta(\beta) \\
& =\Theta(\beta)[\nu I-A]
\end{aligned}
$$

For $\gamma=e_{0}+\left(\nu-\omega_{1}\right) \int_{0}^{t} \exp \left(-\omega_{1} s\right) d s \in \subseteq(\omega)$, we have

$$
f_{\gamma}(\lambda)=\int_{0}^{\infty} \exp (\lambda t) d \gamma=1+\left(\nu-\omega_{1}\right)\left(\omega_{1}-\lambda\right)^{-1}=(\nu-\lambda)\left(\omega_{1}-\lambda\right)^{-1} .
$$

Thus $\Theta(\gamma)=I+\left(\nu-\omega_{1}\right) R\left(\omega_{1} ; A\right)=(\nu I-A) R\left(\omega_{1} ; A\right)$. Further

$$
f_{\beta \gamma}(\lambda)=f_{\beta}(\lambda) f_{\gamma}(\lambda)=\left[f_{\alpha}(\nu)-f_{\alpha}(\lambda)\right]\left(\omega_{1}-\lambda\right)^{-1}
$$

from which it follows that $\beta \gamma=\left[f_{\alpha}(\nu) e_{0}-\alpha\right] * \int_{0}^{t} \exp \left(-\omega_{1} s\right) d s\left({ }^{9}\right)$. Hence

$$
\begin{aligned}
(\nu I-A) \Theta(\beta) R\left(\omega_{1} ; A\right) & =\dot{\Theta}(\beta)(\nu I-A) R\left(\omega_{1} ; A\right)=\Theta(\beta \gamma) \\
& =\left[f_{\alpha}(\nu) I-\Theta(\alpha)\right] R\left(\omega_{1} ; A\right) .
\end{aligned}
$$

Since the range of $R\left(\omega_{1} ; A\right)$ is precisely $\mathfrak{D}(A)$, the second equation of (23) follows directly. On the other hand, $\mathfrak{D}(A)$ is dense in $\mathfrak{X}$, so that given $x_{0} \in \mathfrak{X}$ there exist $x_{n} \in \mathfrak{D}(A)$ such that $x_{n} \rightarrow x_{0}$. Now both $\Theta(\beta) x_{n}$ and $\left[f_{\alpha}(\nu) I-\Theta(\alpha)\right] x_{n}$ converge to limits and, since $A$ is closed, it follows that $(\nu I-A) \Theta(\beta) x_{0}$ $=\left[f_{\alpha}(\nu) I-\Theta(\alpha)\right] x_{0}$. The first part of our theorem then follows immediately from (23).

$\left({ }^{9}\right)$ We use $*$ to indicate the convolution. 
To prove the converse, suppose that $\mu \in \Sigma[\Theta(\alpha)] \quad(\mu \neq \alpha(0))$. Now $\lim _{|\lambda| \rightarrow \infty} f_{\alpha}(\lambda)=\alpha(0)$ uniformly with respect to $\lambda$ in every sector $\pi / 2+\epsilon$ $\leqq$ arg $\lambda \leqq 3 \pi / 2-\epsilon$. Hence by Lemma 5.1, $f_{\alpha}(\lambda)=\mu$ can have at most a finite number of roots in $\Sigma(A)$, say $\nu_{1}, \nu_{2}, \cdots, \nu_{n}$. By equation (21), $\mu \in f_{\alpha}[\Sigma(A)]$. Hence there is at least one root. We now set $Q(\lambda)=\prod_{k=1}^{n}\left(\nu_{k}-\lambda\right)$ and define

$$
f_{\beta}(\lambda)=\left[\mu-f_{\alpha}(\lambda)\right]\left(\omega_{1}-\lambda\right)^{n} / Q(\lambda) .
$$

If we split $\left(\omega_{1}-\lambda\right)^{n} / Q(\lambda)$ into partial fractions, $f_{\beta}(\lambda)$ becomes a sum of terms of the type $f_{\beta_{j}}(\lambda)=\left[\mu-f_{\alpha}(\lambda)\right][\nu-\lambda]^{-j}$. For $j=1$ we showed above that $\beta_{1} \in \mathfrak{S}\left(\omega^{\prime \prime}\right)$ where $\omega^{\prime \prime}(t)=\max \left[\omega(t), \omega_{2} t\right]$ and $\omega_{0}<\omega_{2}<\omega_{1}$. For $j=2, f_{\beta_{1}}(\nu)=0$ and hence $f_{\beta_{2}}(\lambda)=\left[f_{\beta_{1}}(\nu)-f_{\beta_{1}}(\lambda)\right](\nu-\lambda)^{-1}$ so that the same argument proves that $\beta_{2} \in \mathbb{S}\left(\omega^{\prime \prime \prime}\right)$ where $\omega^{\prime \prime \prime}(t)=\max \left[\omega(t), \omega_{3} t\right]$ and $\omega_{0}<\omega_{3}<\omega_{2}$. It follows by induction that $\beta \in S(\omega)$. We see from (24) that $f_{\beta}(\lambda)$ is different from zero in $\Sigma(A)$ and that $\beta(0)=\mu-\alpha(0) \neq 0$. Hence $\Theta(\beta)\left(m^{\prime}\right) \neq 0$ on $\mathfrak{M}^{\prime}=\mathfrak{W}^{\prime} \cup \mathfrak{Z}^{\prime}$. By the Gelfand theory, $B=\Theta(\beta)^{-1}$ exists in $\Re$. Again as before for $f_{\gamma}(\lambda)$ $=Q(\lambda) /\left(\omega_{1}-\lambda\right)^{n}, \quad \gamma \in \subseteq(\omega)$ and $\Theta(\gamma)=Q(A) R\left(\omega_{1} ; A\right)^{n}$. Finally $f_{\beta \gamma}(\lambda)$ $=f_{\beta}(\lambda) f_{\gamma}(\lambda)=\mu-f_{\alpha}(\lambda)$ from which it follows that

$$
\Theta(\beta) Q(A) R\left(\omega_{1} ; A\right)^{n}=\mu I-\Theta(\alpha),
$$

and hence

$$
Q(A) R\left(\omega_{1} ; A\right)^{n}=B[\mu I-\Theta(\alpha)]=[\mu I-\Theta(\alpha)] B .
$$

If we set $O_{k}(A)=\left(\nu_{k} I-A\right) R\left(\omega_{1} ; A\right)$, then (25) becomes

$$
\prod_{k=1}^{n} O_{k}(A)=B[\mu I-\Theta(\alpha)]=[\mu I-\Theta(\alpha)] B .
$$

It should be noted that

$$
O_{k}(A)=\left[\left(\nu_{k}-\omega_{1}\right) I+\left(\omega_{1} I-A\right)\right] R\left(\omega_{1} ; A\right)=\left(\nu_{k}-\omega_{1}\right) R\left(\omega_{1} ; A\right)+I
$$

is a bounded operator. Suppose now that $[\mu I-\Theta(\alpha)]$ has $\mathrm{P}_{1}$. Then by (26) one of the $O_{k}(A)$ has $\mathrm{P}_{1}$ and since $R\left(\omega_{1} ; A\right)$ does not, the corresponding $\left(\nu_{k} I-A\right)$ must have $\mathrm{P}_{1}$. If $[\mu I-\Theta(\alpha)]$ has $\mathrm{P}_{2}$, then the range of $\prod_{k=1}^{n} O_{k}(A)$ is not dense in $\mathfrak{X}$. If the range of $\prod_{k=2}^{n} O_{k}(A)$ is dense in $\mathfrak{X}$, then the range of $O_{1}(A)$ could not be dense in $\mathfrak{X}$ since otherwise $\prod_{k=2}^{n} O_{k}(A)$, being bounded, would take a dense set again into a dense set. If, on the other hand, $\prod_{k=2}^{n} O_{k}(A)$ does not have a dense range, we proceed by induction and find some $O_{k}(A)$ with a nondense range. Since the range of $R\left(\omega_{1} ; A\right)$ is precisely $\mathfrak{D}(A),\left(\nu_{k} I-A\right)$ must then have $\mathrm{P}_{2}$. Finally if $[\mu I-\Theta(\alpha)]$ has $\mathrm{P}_{3}$ but neither $\mathrm{P}_{1}$ nor $\mathrm{P}_{2}$, then since the nonvacuous set $\left\{\nu_{k}\right\} \subset \Sigma(A)$ and since none of the $\left[\nu_{k} I-A\right]$ can have $\mathrm{P}_{1}$ or $\mathrm{P}_{2}$, it follows by the first part of the theorem that at least one $\nu_{k}$ must have $\mathrm{P}_{3}$.

Before concluding this section, two remarks are in order. In the first place we cannot hope for a classification of $T(t)$ continuous in the uniform topology 
for $t \geqq c>0$ on the basis of the decomposition of $\mathfrak{M}^{\prime}$ alone as was possible for $T(t)$ uniformly continuous at $t=0$. This follows from the existence of semigroups of operators strongly continuous but nowhere uniformly continuous for which $T(t)$ is quasi-nilpotent for all $t>0$ (see for example Hille [6, section 16.4]). In this case of course $\mathfrak{M}^{\prime} \equiv \mathfrak{Z}^{\prime}$.

For $T(t)$ continuous in the uniform topology $(t \geqq c>0)$ we have

$$
\lim _{t \rightarrow \infty} t^{-1} \log \|T(t)\|=\log \|T(1)\| \|=\sup R[\Sigma(A)] .
$$

Hence if we set $c(x)$ equal to the abscissa of convergence for $R(\lambda ; A) x$ $=\int_{0}^{\infty} \exp (-\lambda t) T(t) x d t$, then $\sup c(x)=\lim _{t \rightarrow \infty} t^{-1} \log \|T(t)\|$. It is not known whether this result holds in general for $T(t)$ strongly continuous on $(0, \infty)$.

6. Analytical semi-groups. The basic material on analytical semi-groups is to be found in Hille [6, Chap. 13]. We shall limit ourselves to some generalizations of Hille's work on the rate of growth of $T(\zeta)$. These results follow quite naturally from our approach, whereas the Laplace transform methods used by Hille do not seem to yield them.

Definition. An essentially angular semi-module $\Gamma$ is by definition an open simply-connected semi-module $\left(\zeta_{1}, \zeta_{2} \in \Gamma \cdot \supset \cdot \zeta_{1}+\zeta_{2} \in \Gamma\right)$ having two asymptotic angles $\phi_{1}, \phi_{2}\left(-\pi / 2 \leqq \phi_{1}<0<\phi_{2} \leqq \pi / 2\right)$ such that for each $\epsilon>0$ there exists an $r_{\epsilon}$ for which the infinite sector

$$
\phi_{1}+\epsilon \leqq \arg \zeta \leqq \phi_{2}-\epsilon, \quad|\zeta| \geqq r_{\epsilon},
$$

lies entirely within $\Gamma . \Gamma$ does not contain the origin.

THEOREM 6.1. Let the semi-group of operators $T(\zeta)$ be defined and holomorphic in the essentially angular semi-module $\Gamma$. Define the indicator

$$
\sigma(\phi)=\lim _{r \rightarrow \infty} r^{-1} \log \|T[r \exp (i \phi)]\| \quad \text { for } \phi_{1}<\phi<\phi_{2} .
$$

Then either (a) $\sigma(\phi) \equiv-\infty$ in which case $T(\zeta)$ is a quasi-nilpotent element of $\mathbb{E}(\mathfrak{X})$ for all $\zeta \in \Gamma$; or else (b) $\sigma(\phi)$ is always finite-valued in which case $\sigma(\phi)$ is the function of support for a closed convex set $\mathfrak{D}$ which will be called the indicator diagram for the semi-group $T(\zeta)$.

We consider a commutative ring $\Re^{\prime}$ containing the operators $[T(\zeta) \mid \zeta \in \Gamma]$ and the identity $I$. Let $\mathfrak{M}^{\prime}$ be the set of all maximal ideals $m^{\prime}$ in $\Re^{\prime}$. By the Gelfand theory [3], $T(\zeta)$ has a representation as a continuous function $T(\zeta)\left(m^{\prime}\right)$ in $\mathfrak{M}^{\prime}$. Since $T(\zeta)$ is holomorphic in $\Gamma$, so likewise is $T(\zeta)\left(m^{\prime}\right)$ for each $m^{\prime}$. By the semi-group property

$$
T\left(\zeta_{1}+\zeta_{2}\right)\left(m^{\prime}\right)=T\left(\zeta_{1}\right)\left(m^{\prime}\right) \cdot T\left(\zeta_{2}\right)\left(m^{\prime}\right) \quad \text { for } \zeta_{1}, \zeta_{2} \in \Gamma .
$$

The only such complex-valued function holomorphic in the simply-connected semi-module $\Gamma$ is either identically zero or else of the form exp $\left[a\left(m^{\prime}\right) \zeta\right]$. In conformity with our previous notation we shall say that $m^{\prime} \in Z^{\prime}$ if $T(\zeta)\left(m^{\prime}\right)$ 
$\equiv 0$ and $m^{\prime} \in \mathfrak{W}^{\prime}$ otherwise.

Now the limit in (27) is shown to exist by the familiar argument (see $[6$, p. 135]). Hence

$$
\sigma(\phi)=\lim _{n \rightarrow \infty}(r n)^{-1} \log \left\|T[r \exp (i \phi)]^{n}\right\|=r^{-1} \log \|T[r \exp (i \phi)]\| .
$$

In other words

$$
\sigma(\phi)=\sup \left[r^{-1} \log \left|T[r \exp (i \phi)]\left(m^{\prime}\right)\right| m^{\prime} \in \mathfrak{M}^{\prime}, r \exp (i \phi) \in \Gamma\right] .
$$

If $\mathfrak{M}^{\prime} \equiv \mathfrak{Z}^{\prime}$, then $T(\zeta)\left(m^{\prime}\right) \equiv 0$ and $\sigma(\phi) \equiv-\infty$; in this case each $T(\zeta)$ is quasinilpotent. On the other hand if $\mathfrak{B}^{\prime}$ is nonvacuous, then $T(\zeta)\left(m^{\prime}\right)$ $=\exp \left[a\left(m^{\prime}\right) \zeta\right]$ in $\mathfrak{S}^{\prime}$ and

$$
\sigma(\phi)=\sup \left[R\left[a\left(m^{\prime}\right) \exp (i \phi)\right] \mid m^{\prime} \in \mathfrak{W}^{\prime}\right] .
$$

Thus $\sigma(\phi)$ is the supremum of the projections of $a\left(m^{\prime}\right)^{*}$ on the unit vector $\exp (i \phi)\left({ }^{10}\right)$. Thus if we set

$$
\mathfrak{D}=\text { closed convex extension of }\left[a\left(m^{\prime}\right)^{*} \mid m^{\prime} \in \mathfrak{W}^{\prime}\right],
$$

then $\sigma(\phi)$ is the function of support for $\mathfrak{D}$.

It is customary to define an extremal point of a closed convex point set $\mathfrak{D}$ as a point on the boundary of $\mathfrak{D}$ which is not an interior point of any line segment belonging to the boundary of $\mathfrak{D}$. An extremal point $\lambda_{0}$ is called exceptional if it is the end point of a line segment on the boundary of $D$ and the extension of this line segment is the only line of support of $\mathfrak{D}$ passing through $\lambda_{0}$. It is clear that every exceptional extremal is the limit of ordinary extremal points.

We now seek to relate the indicator diagram $\mathfrak{D}$ to the spectrum of the infinitesimal generator $A$. It is clear that even to define $A$ we must impose further conditions on $T(\zeta)$ near the origin.

THEOREM 6.2. Let $T(\zeta)$ be a semi-group of linear transformations of type $(\mathrm{H})$ on $[0, \infty)$ and holomorphic on the essentially angular semi-module $\Gamma$. Then the indicator diagram $\mathfrak{D}=$ closed convex extension $\Sigma(A)^{*} . R(\lambda ; A)$ exists and is holomorphic outside of $\mathfrak{D}^{*}$ and every extremal point of $\mathfrak{D}^{*}$ is a singular point of $R(\lambda ; A)$. Finally if $\sigma(\phi) \equiv-\infty$ in $\left(\phi_{1}, \phi_{2}\right)$, then $R(\lambda ; A)$ is an entire function of $\lambda$.

We have merely to replace $\Re^{\prime}$ by the ring $R$ defined in section three. Since $T(\zeta)$ is continuous in the uniform topology for $\xi \geqq 0(\zeta=\xi+i \eta)$ and sufficiently large, it follows that the subdivision of $\mathfrak{M}^{\prime}$ into $\mathfrak{B}^{\prime}$ and $\mathfrak{Z}^{\prime}$ which we made in the proof of Theorem 6.1 agrees with that which we previously made. By Theorem 3.1, $\Sigma(A)=\left[a\left(m^{\prime}\right) \mid m^{\prime} \in \mathfrak{B}^{\prime}\right]$ and therefore $\mathfrak{D}=$ closed convex extension of $\Sigma(A)^{*}$. Now $R(\lambda ; A)$ is holomorphic outside of $\Sigma(A)$ and a

(10) We denote the complex conjugate of $a$ by $a^{*}$. 
fortiori outside of $\mathfrak{D}^{*}$. To show that every extremal point of $\mathfrak{D}^{*}$ is a singular point of $R(\lambda ; A)$ it is clearly enough to do this for ordinary extremal points. However if an ordinary extremal point $\lambda_{0}$ were a regular point for $R(\lambda ; A)$, then we could separate a small neighborhood of $\lambda_{0}$ from the rest of $\mathfrak{D}^{*}$ by a suitable parallel to one of the lines of support of $\mathfrak{D}^{*}$ through $\lambda_{0}$ in such a manner that the part of $\mathfrak{D}^{*}$ cut off by this parallel lies in this neighborhood. In this case, however, $\lambda_{0}$ would not be a boundary point of the closed convex extension of $\Sigma(A)$, contrary to our choice of $\lambda_{0}$.

7. Semi-groups in Hilbert space. If the semi-group of operators can be embedded in a commutative self-adjoint algebra $\Re$ of operators on a Hilbert space, then it is possible to obtain exceedingly precise results out of the representation theorem for $\Re$. One can, in fact, derive directly the spectral resolution theorems for semi-groups of normal operators due originally to Stone [11], Sz. Nagy [8], and Hille [6]. For purposes of illustration we shall obtain the spectral resolution theorem for a group of unitary operators where the group involved is any locally compact abelian group (5). Stone first discovered this result for the additive group of real numbers and this was later generalized by Ambrose [1] and Neumark [9] to the locally compact abelian groups.

We assume that we are given a strongly continuous unitary representation $U(g)$ of the group (s). That is

(a) $U(g)$ is a unitary operator in the Hilbert space $\mathfrak{X}$,

(b) $U(g) x$ is continuous on $\&$ in the Hilbert space metric,

(c) $U\left(g_{1}+g_{2}\right)=U\left(g_{1}\right) U\left(g_{2}\right)$.

We now set $\Re$ equal to the strong closure of the set of all polynomials in $[U(g) \mid g \in \mathbb{B}]$. Then $\Re$ is isomorphic and isometric with the ring $\mathfrak{C}^{*}(\mathfrak{M})$ of all continuous complex-valued functions defined on a totally disconnected compact Hausdorff space $\mathfrak{M}$ of maximal ideals $m$ in $\Re$ (see [12], [13], [14], and [16]). The clopen (closed and open) subsets of $\mathfrak{M}$ are in one-to-one correspondence with the projection operators in $\Re$, and this correspondence is such that if $\sigma \leftrightarrow E(\sigma)$, then $E(\sigma)(m)$ is the characteristic function of the clopen set $\sigma$. Further, the closure of any open set is clopen. Thus each open set differs from a unique clopen set by a set of the first category. From this it follows that to each Borel measurable subset $\sigma$ of $\mathfrak{M}$ there corresponds a unique clopen set $\Psi(\sigma)$ which differs from $\sigma$ only on a set of the first category. The projection operators $E[\Psi(\sigma)]$ over the Borel measurable subsets of $\mathfrak{M}$ have the following properties:

(a) If $\sigma_{1} \cap \sigma_{2}=\phi$, then $E\left[\Psi\left(\sigma_{1}\right)\right] E\left[\Psi\left(\sigma_{2}\right)\right]=0$.

(b) $E[\Psi(\mathfrak{M})]=I$ and $E[\Psi(\phi)]=0$.

(c) If $\sigma_{i} \cap \sigma_{j}=\phi$ for $i \neq j$, then $E\left[\Psi\left(U \sigma_{i}\right)\right]=\sum_{i=1}^{\infty} E\left[\Psi\left(\sigma_{i}\right)\right]$ where the partial sums converge in the strong operator topology.

In other words $E[\Psi(\sigma)]$ is a resolution of the identity relative to the Borel measurable subsets of $\mathfrak{M}$ 
THEOREM 7.1. Let $U(g)$ be a strongly continuous unitary representation of the locally compact abelian group (\$). Then there exists a resolution of the identity $F\left(\sigma^{\prime}\right)$ relative to the Borel measurable subsets of the character group $\& \mathbb{S}^{*} \equiv[\chi(g)]$ such that

$$
U(g)=\int_{\mathscr{S} *} \chi(g) d F\left(\sigma^{\prime}\right)
$$

where the integral converges in the uniform operator topology.

It is clear for a fixed $m$ that $U\left(g_{1}+g_{2}\right)(m)=U\left(g_{1}\right)(m) U\left(g_{2}\right)(m)$ and $|U(g)(m)| \leqq\|U(g)\|=1$ and hence that $U(g)(m)$ is a character of the group (5) However, $U(g)(m)$ need not be a continuous character. We shall show that, except for a set of the first category in $\mathfrak{M}, U(g)(m)$ will be a continuous character. To this end we consider the class $\mathfrak{R}$ of Lebesgue summable functions $w(g)$ on $\&$ (relative to the Haar measure $d g$ ). Since $U(g)$ is strongly continuous, the Bochner integral $\int w(g) U(g) x d g$ exists for each $x \in \mathfrak{X}$ and defines a bounded linear operator $A_{w}$ belonging to $\mathfrak{R}$. We now split $\mathfrak{M}$ into two sets:

(a) $\mathfrak{B}: m_{0}$ belongs to $\mathfrak{B}$ if there exists a $w_{0} \in \mathfrak{R}$ for which $A_{w_{0}}\left(m_{0}\right) \neq 0$.

(b) $\mathfrak{U}: m_{0}$ belongs to $\mathfrak{U}$ if $A_{w}\left(m_{0}\right)=0$ for all $w \in \mathfrak{R}$.

We see that $\mathfrak{W} \cup \mathfrak{U}=\mathfrak{M}$ and that $\mathfrak{W} \cap \mathfrak{U}=\phi$. Further $\mathfrak{B}$ is an open subset of $\mathfrak{M}$ since it is the union of the open sets $\left[m \mid A_{w}(m) \neq 0\right]$ over all $w \in \mathbb{R}$.

If $m_{0} \in \mathfrak{B}$, then $U(g)\left(m_{0}\right)$ is a continuous character. For let $w_{0} \in \mathfrak{R}$ be such that $A_{w_{0}}\left(m_{0}\right) \neq 0$. Then

$$
U(h) A_{w_{0}}=\int U(h+g) w_{0}(g) d g=\int U(g) w_{0}(g-h) d g
$$

and hence

$$
\left\|U\left(h_{1}\right) A_{w_{0}}-U\left(h_{2}\right) A_{w_{0}}\right\| \leqq \int\|U(g)\|\left|w_{0}\left(g-h_{1}\right)-w_{0}\left(g-h_{2}\right)\right| d g .
$$

It follows that $U(h) A_{w_{0}}$ is continuous in the uniform operator topology and a fortiori that $U(h)\left(m_{0}\right) A_{w_{0}}\left(m_{0}\right)=\left[U(h) A_{w_{0}}\right]\left(m_{0}\right)$ is continuous. Since $A_{w_{0}}\left(m_{0}\right)$ $\neq 0$, we have that $U(h)\left(m_{0}\right)$ is itself continuous. If $m_{0} \in \mathfrak{B}$ we can obtain the further result that $A_{w}\left(m_{0}\right)=\int U(g)\left(m_{0}\right) w(g) d g$ for all $w \in \mathbb{R}$. For since $U(h) A_{w_{0}}$ is continuous in the uniform operator topology, the integral $\int w(h) U(h) A_{w_{0}} d h$ will converge in this topology. Hence the multiplicative linear functionals commute with this integration. On the other hand

$$
\int w(h) U(h) A_{w_{0}}(x) d h=A_{w}\left[A_{w_{0}}(x)\right] .
$$

Combining these two facts, we get 


$$
\begin{aligned}
A_{w}\left(m_{0}\right) A_{w_{0}}\left(m_{0}\right) & =\left[A_{w} A_{w_{0}}\right]\left(m_{0}\right)=\int w(h)\left[U(h) A_{w_{0}}\right]\left(m_{0}\right) d h \\
& =\int w(h) U(h)\left(m_{0}\right) A_{w_{0}}\left(m_{0}\right) d h=A_{w_{0}}\left(m_{0}\right) \int w(h) U(h)\left(m_{0}\right) d h .
\end{aligned}
$$

The result now follows from the fact that $A_{w_{0}}\left(m_{0}\right) \neq 0$.

We shall now show that $\overline{\mathfrak{B}}=\mathfrak{M}$. Suppose the contrary were so. Then $\mathfrak{W}$ being open implies that $\overline{\mathfrak{W}}$ is clopen and hence that $\sigma=\mathfrak{M}-\overline{\mathfrak{W}}$ is clopen. Clearly $E(\sigma)(m)=0$ on $\mathfrak{W}$. On the other hand let $\mathfrak{B}$ be a neighborhood of the identity in $\mathfrak{B}$ with finite measure $c_{\mathfrak{b}}$. We define $w_{\mathfrak{b}}=c_{\mathfrak{b}}^{-1}$ on $\mathfrak{B}$ and zero elsewhere. The neighborhoods $\mathfrak{B}$ form a directed set $\left(\mathfrak{B}_{2} \geqq \mathfrak{B}_{1}\right.$ means that $\left.\mathfrak{B}_{2} \subset \mathfrak{B}_{1}\right)$ and since $U(g)$ is strongly continuous, $\lim _{\mathfrak{v}} A_{w_{\mathfrak{v}}}(x)=x$ for each $x \in \mathfrak{X}$. Finally since $w_{\mathfrak{v}} \in \mathbb{R}, A_{w_{\mathfrak{b}}}(m)=0$ on $\mathfrak{U} \supset \sigma$. Hence $E(\sigma)(m) A_{w_{\mathfrak{b}}}(m) \equiv 0$ on $\mathfrak{M}$ so that $E(\sigma) A_{w_{\mathfrak{v}}}=0$. But then

$$
0=\left(E(\sigma) A_{w_{\mathfrak{v}}} x, x\right)=\left(A_{w_{\mathfrak{v}}} x, E(\sigma) x\right) \rightarrow(x, E(\sigma) x)
$$

for all $x \in \mathfrak{X}$ and hence $E(\sigma)=0$ contrary to assumption. This incidently shows that $\mathfrak{M}-\mathfrak{W}$ is of the first category and also that $\Psi(\mathfrak{W})=\mathfrak{M}$.

We next define a mapping $\Phi$ on $\mathfrak{W}$ into $\mathfrak{H}^{*}$ : each $m \in \mathfrak{W}$ maps into the continuous character $\chi(g) \equiv U(g)(m)$. In order to show that $\Phi$ is a continuous mapping it is convenient to introduce the equivalent neighborhood system for $\mathfrak{S}^{*}$ defined by $\epsilon>0$ and finite subsets $w_{1}, w_{2}, \cdots, w_{n}$ of $\mathfrak{R}$ as

$$
\mathfrak{N}^{\prime}\left(\chi_{0}\right) \equiv\left[\chi \| \int \chi(g) w_{i}(g) d g-\int \chi_{0}(g) w_{i}(g) d g \mid<\epsilon ; i=1,2, \cdots, n\right] .
$$

It is clear for $\chi_{0}=\Phi\left(m_{0}\right)$ for $m_{0} \in \mathfrak{W}$ that

$$
\mathfrak{N}\left(m_{0}\right) \equiv\left[m|| A_{w_{i}}(m)-A_{w_{i}}\left(m_{0}\right) \mid<\epsilon ; i=1,2, \cdots, n ; m \in \mathfrak{W}\right]
$$

maps into $\mathfrak{N}^{\prime}\left(\chi_{0}\right)$. Since $\Phi$ is continuous on $\mathfrak{W}$ into $\mathfrak{H}^{*}$, and since $\mathfrak{W}$ is an open subset of $\mathfrak{M}$, it follows that $\Phi^{-1}$ maps open sets in $\mathfrak{B}^{*}$ into open subsets of $\mathfrak{M}$, closed sets in $\mathfrak{S H}^{*}$ into the complements of open subsets of $\mathfrak{M}$ relative to $\mathfrak{W}$; and more generally Borel subsets of $\mathfrak{S}^{*}$ into Borel subsets of $\mathfrak{M}$.

We are now essentially through. For given a Borel measurable subset $\sigma^{\prime}$ of $\mathfrak{S}^{*}, \Phi^{-1}\left(\sigma^{\prime}\right)$ is a Borel measurable subset of $\mathfrak{M}$; and we can define

$$
F\left(\sigma^{\prime}\right)=E\left[\Psi\left(\Phi^{-1}\left(\sigma^{\prime}\right)\right)\right] \text {. }
$$

From what we have said earlier it is clear that $F\left(\sigma^{\prime}\right)$ is a completely additive (in the strong topology) resolution of the identity. Finally for a given $g \in(5$, let $\left\{\sigma_{1}^{\prime}, \sigma_{2}^{\prime}, \cdots, \sigma_{n}^{\prime}\right\}$ be a subdivision of ()$^{*}$ into disjoint Borel sets such that for some numbers $\left\{a_{1}, a_{2}, \cdots, a_{n}\right\}$ we have $\left|\chi(g)-a_{i}\right| \leqq \epsilon$ for all $\chi \in \sigma_{i}^{\prime}$. By the definition of $\Phi$ it follows that $\left|U(g)(m)-a_{i}\right| \leqq \epsilon$ for all $m \in \Phi^{-1}\left(\sigma_{i}^{\prime}\right)$, and hence for all $m \in \Psi\left[\Phi^{-1}\left(\sigma_{i}^{\prime}\right)\right]$ except perhaps for a set of the first category. Thus, except for a set of the first category, 


$$
\left|U(g)(m)-\sum_{i=1}^{n} a_{i} F\left(\sigma_{i}^{\prime}\right)(m)\right| \leqq \epsilon\left({ }^{11}\right) .
$$

On the other hand, both $U(g)(m)$ and $\sum_{i=1}^{n} a_{i} F\left(\sigma_{i}^{\prime}\right)(m)$ are continuous functions on $\mathfrak{M}$ and since they can differ by more than $\epsilon$ only on a set of the first category, it follows that they can nowhere differ by more than $\epsilon$. In other words $\left\|U(g)-\sum_{i=1}^{n} a_{i} F\left(\sigma_{i}^{\prime}\right)\right\| \leqq \epsilon$. Finally since $\chi(g)$ is Borel measurable on (5)*, we have $U(g)=\int_{\circlearrowleft *} \chi(g) d F\left(\sigma^{\prime}\right)$, where the integral converges in the uniform operator topology.

\section{REFERENCES}

1. Warren Ambrose, Spectral resolution of groups of unitary operators, Duke Math. J. vol. 11 (1944) pp. 589-595.

2. A. Beurling, Sur les intégrales de Fourier absolument convergentes et leur application à une transformation fonctionelle, Neuvième Congrès Math. Scand. Helsingfors, 1938, pp. 345-366.

3. I. Gelfand, Normierte Ringe, Rec. Math. (Mat. Sbornik) N.S. vol. 9 (1941) pp. 3-24.

4. - Zur Theorie der Charaktere der Abelschen topologischen Gruppen, Rec. Math. (Mat. Sbornik) N.S. vol. 9 (1941) pp. 49-50.

5. - U Über absolut konvergente trigonometrische Reihen und Integrale, Rec. Math. (Mat. Sbornik) N.S. vol. 9 (1941) pp. 51-66.

6. Einar Hille, Functional analysis and semi-groups, Amer. Math. Soc. Colloquium Publications, vol. 31, New York, 1948.

7. - On the differentiability of semi-group operators, Acta Univ. Szeged. vol. 12 (1950) part B, pp. 19-24.

8. B. v. Sz. Nagy, Spektraldarstellung linearer Transformationen des Hilbertschen Raumes, Ergebnisse der Mathematik, vol. 5, Berlin, 1942.

9. M. Neumark, Positive definite operator functions on a commutative group, Bull. Acad. Sci. URSS. vol. 7 (1943) pp. 237-244.

10. R. S. Phillips, On semi-groups of operators, Proceedings of the American Mathematical Society vol. 2 (1951) pp. 234-237.

11. M. Stone, On one parameter unitary groups in Hilbert spaces, Ann. of Math. vol. 33 (1932) pp. 643-648.

12. - - A general theory of spectra I, Proc. Nat. Acad. Sci. U.S.A. vol. 26 (1940) pp. 280-283.

13. - , A general theory of spectra II, Proc. Nat. Acad. Sci. U.S.A. vol. 27 (1941) pp. 83-87.

14. - Boundedness properties in function-lattices, Canadian Journal of Mathematics vol. 1 (1949) pp. 176-186.

15. - On a theorem of Pólya, J. Indian Math. Soc. N.S. vol. 12 (1948) pp. 1-7.

16. I. Vernikoff, S. Krein, and A. Tovbin, Sur les anneaux semi-ordonnés, C. R. (Doklady) Acad. Sci. URSS. N.S. vol. 30 (1941) pp. 785-787.

Institute for Advanced Study,

Princeton, N. J.

University of Southern California,

Los Angeles, Calif.

(11) Note that for each $m \in \mathfrak{M}$, only one of the $F\left(\sigma_{i}^{\prime}\right)(m)$ has the value one, the remainder are zero-valued. 